BNL-114810-2017-JA

\title{
Injectable Anisotropic Nanocomposite Hydrogels Direct In Situ Growth and Alignment of Myotubes
}

\author{
Kevin J. De France, Kevin G. Yager, Katelyn J.W. Chan, \\ Brandon Corbett, Emily D. Cranston, and Todd Hoare
}

Submitted to Nano Letters

September 2017

Center for Functional Nanomaterials

Brookhaven National Laboratory

\begin{abstract}
U.S. Department of Energy USDOE Office of Science (SC), Basic Energy Sciences (SC-22)
\end{abstract}

Notice: This manuscript has been authored by employees of Brookhaven Science Associates, LLC under Contract No. DE- SC0012704 with the U.S. Department of Energy. The publisher by accepting the manuscript for publication acknowledges that the United States Government retains a non-exclusive, paid-up, irrevocable, world-wide license to publish or reproduce the published form of this manuscript, or allow others to do so, for United States Government purposes. 


\section{DISCLAIMER}

This report was prepared as an account of work sponsored by an agency of the United States Government. Neither the United States Government nor any agency thereof, nor any of their employees, nor any of their contractors, subcontractors, or their employees, makes any warranty, express or implied, or assumes any legal liability or responsibility for the accuracy, completeness, or any third party's use or the results of such use of any information, apparatus, product, or process disclosed, or represents that its use would not infringe privately owned rights. Reference herein to any specific commercial product, process, or service by trade name, trademark, manufacturer, or otherwise, does not necessarily constitute or imply its endorsement, recommendation, or favoring by the United States Government or any agency thereof or its contractors or subcontractors. The views and opinions of authors expressed herein do not necessarily state or reflect those of the United States Government or any agency thereof. 


\section{Injectable Anisotropic Nanocomposite Hydrogels}

\section{Direct In Situ Growth and Alignment of}

\section{Myotubes}

Kevin J. De France, ${ }^{\dagger}$ Kevin G. Yager, ${ }^{,}$Katelyn J.W. Chan, ${ }^{\dagger}$ Brandon Corbett, ${ }^{\dagger}$ Emily D.

$$
\text { Cranston, }{ }^{\dagger} \text { and Todd Hoare }{ }^{\dagger}, *
$$

$\dagger$ Department of Chemical Engineering, McMaster University, 1280 Main Street West, Hamilton, ON L8S 4L8, Canada

\$ Center for Functional Nanomaterials, Brookhaven National Laboratory,

Upton, NY, 11973, USA 
ABSTRACT. While injectable in situ crosslinking hydrogels have attracted increasing attention as minimally invasive tissue scaffolds and controlled delivery systems, their inherently disorganized and isotropic network structure limits their utility in engineering oriented biological tissues. Traditional methods to prepare anisotropic hydrogels are not easily translatable to injectable systems given the need for external equipment to direct anisotropic gel fabrication and/or the required use of temperatures or solvents incompatible with biological systems. Herein, we report a new class of injectable nanocomposite hydrogels based on hydrazone crosslinked poly(oligoethylene glycol methacrylate) (POEGMA) and magnetically-aligned cellulose nanocrystals (CNCs) capable of encapsulating skeletal muscle myoblasts and promoting their differentiation into highly oriented myotubes in situ. CNC alignment occurs on the same timescale as network gelation and remains fixed after the removal of the magnetic field, enabling concurrent $\mathrm{CNC}$ orientation and hydrogel injection. The aligned hydrogels show mechanical and swelling profiles that can be rationally modulated by the degree of $\mathrm{CNC}$ alignment and can direct myotube alignment both in $2 \mathrm{D}$ and $3 \mathrm{D}$ following co-injection of the myoblasts with the gel precursor components. As such, these hydrogels represent a critical advancement in anisotropic biomimetic scaffolds that can be generated non-invasively in vivo following simple injection.

Keywords: muscle tissue engineering, cellulose nanocrystals, anisotropic hydrogels, injectable hydrogels, magnetic alignment, nanocomposite biomaterials 
Table of Contents Graphic
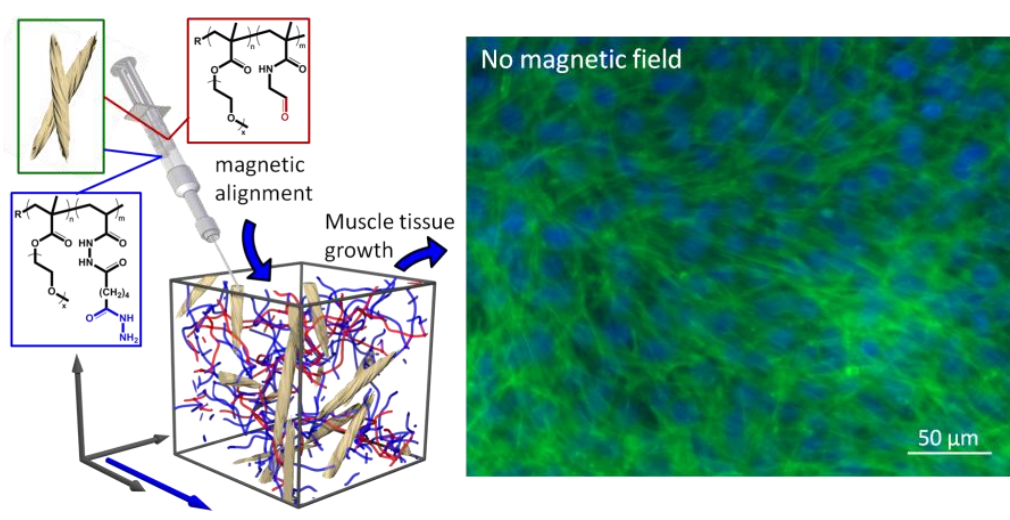

$0.56 \mathrm{~T}$ magnetic field

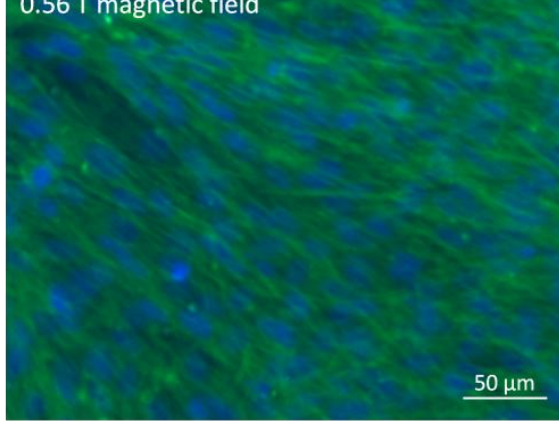


Injectable hydrogels combine many of the advantages associated with traditional bulk hydrogels (e.g., high swellability, controllable nanoporosity, ease of functionalization, and high cytocompatibility) with the added benefit of minimally invasive delivery. ${ }^{1-4}$ This combination of properties has sparked extensive research into the use of injectable hydrogels for tissue engineering, ${ }^{5,6}$ regenerative medicine, ${ }^{7-9}$ and controlled delivery ${ }^{1,10-13}$ applications. Injectable hydrogels are conventionally formed via physical interactions (e.g., physically entangled or hydrophobically self-associated polymers rendered injectable by shear thinning $)^{14-16}$ or via chemical interactions initiated following an injection-based administration (e.g., in situ gelation of methacrylated pre-polymers using UV irradiation ${ }^{17}$ or gelation of other functional polymers using small molecule crosslinkers ${ }^{18,19}$ ). However, both these strategies have inherent limitations; specifically, the typically low mechanical strength and often rapid dissolution of physicallygelled systems and the potential toxicity of the crosslinking chemistries used for covalentlygelled systems limit the practical utility of such materials. ${ }^{1}$

To address these drawbacks, recent research has focused on the design of thermodynamically (or at least kinetically) orthogonal covalent bond forming chemistries that can form crosslinks upon injection-based mixing, including alkyne-azide reactions, ${ }^{20,21}$ Diels-Alder cycloadditions, ${ }^{22}$ disulfide formation, ${ }^{23,24}$ thiol-Michael additions, ${ }^{25}$ and Schiff base formation. ${ }^{26,27}$ Our group has been particularly active in developing in situ-gelling hydrogels based on poly(oligoethylene glycol methacrylate) (POEGMA) and crosslinked using kinetically bioorthogonal hydrazidealdehyde chemistry. ${ }^{28}$ POEGMA is synthesized through free-radical (co)polymerization; in contrast, poly(ethylene glycol) (the most widely used biomedical hydrogel precursor) is a stepgrowth polymer with limited options for functionalization. Free radical polymerization allows for a significantly higher degree of control over polymer composition, molecular weight, and 
functionality, which in turn enables precise tuning of hydrogel physical properties including gelation time, mechanical strength, degradation, hydrophilicity, thermoresponsiveness, protein adsorption, and cell adhesion. ${ }^{28-34}$

As with all reported injectable hydrogels to-date, the network structure of hydrazone crosslinked POEGMA hydrogels is isotropic. This isotropic network structure is typically not problematic in controlled drug delivery or simple cell encapsulation applications. However, the randomness of the gel microstructure does pose a significant limitation on the use of such gels in tissue engineering and regenerative medicine applications given the hierarchical and anisotropic nature of many biological tissues including muscle, nerve, cornea, and cartilage. ${ }^{35}$ As such, very few reports describing the engineering of anisotropic tissues have used injectable hydrogels as a matrix. Furthermore, to the best of our knowledge, no report has applied an injectable hydrogel to engineer muscle tissue, which ideally requires both a stiffer and hierarchically aligned matrix. ${ }^{36}$ Instead, functional muscle tissue engineering approaches have to-date largely involved either (1) combining muscle cells with a pre-fabricated biomaterial matrix, culturing the cells in vitro, and directly implanting the cell-laden scaffold in vivo, ${ }^{37-43}$ or (2) using a biomaterial scaffold to drive endogenous tissue regeneration by providing a combination of chemical and physical cues. ${ }^{44-47}$ In either case, invasive surgical procedures are required to implant the scaffold within the host, a procedure that could be avoided if minimally-invasive (i.e., injectionbased) delivery methods were developed.

The design of anisotropic and injectable hydrogels has proved challenging, as the various methods to generate anisotropy in vitro (e.g., the use of mechanical force, ${ }^{48-51}$ templated selfassembly ${ }^{52}$ micropatterning/3D printing, ${ }^{53,54}$ or directional freeze-casting ${ }^{55-61}$ ) cannot be directly translated into an injectable system given the need for external equipment and/or the required use 
of temperatures or solvents not compatible with in vivo use. However, given the non-invasive nature of magnetic fields, magnetic alignment does offer promise for the fabrication of injectable anisotropic hydrogel scaffolds. For example, Rose et al. recently reported an injectable fibrinbased hybrid hydrogel incorporating rod-shaped SPION-containing poly(ethylene glycol) microgels which could align in the presence of an external magnetic field, ${ }^{62}$ enabling successful 3D orientation of nerve cells co-delivered with the scaffold. While these matrices display low elastic moduli relevant for neural tissue repair (i.e., spinal cord), the mechanical properties are insufficient for engineering stiffer tissues such as muscle and cartilage. ${ }^{62,63}$

In this work, we utilize a combination of in situ magnetic alignment and reactive coextrusion to orient high aspect ratio cellulose nanocrystals (CNCs) within a nanocomposite POEGMA hydrogel. CNCs are commercially available rigid anisotropic nanoparticles (cross section $\approx 5$ $\mathrm{nm}$, length $\approx 150 \mathrm{~nm}$ ) commonly extracted from cotton or wood pulp through a sulfuric acid hydrolysis process. ${ }^{64-66}$ Previous work in the Eichhorn group showed that mouse myoblasts will preferentially orient on two-dimensional spin-coated CNC thin films in the direction of CNC alignment. $^{67,68}$ When incorporated into hydrogel networks, CNCs have been demonstrated to increase hydrogel strength while also introducing high surface areas, tunable surface chemistries, and a hydrophilic interface for controlling interactions with the gel environment, leading to a variety of functional hydrogel nanocomposites. ${ }^{69}$ Furthermore, due to their highly crystalline structure, CNCs have both a negative diamagnetic anisotropy ${ }^{70,71}$ and a giant dipole moment ${ }^{72}$, making them susceptible to alignment in electromagnetic fields (unlike amorphous polymers like POEGMA). ${ }^{73,74}$ Our recent study on the effects of magnetic field strength and CNC concentration on the alignment kinetics of CNCs in aqueous suspension found that field strengths as low as $0.56 \mathrm{~T}$ enhanced $\mathrm{CNC}$ alignment (both instantaneously and cooperatively 
over time) in a direction perpendicular to the applied magnetic field. ${ }^{74}$ We have also shown that CNC-POEGMA hydrogels can be tailored to gel over time periods ranging from 5 seconds to 25 minutes, and that adsorption of POEGMA to CNCs adds a physical crosslinking component to the hydrogels which improves their mechanical properties by at least one order of magnitude. ${ }^{34}$ Herein, we leverage our capacity to control the kinetics of both CNC alignment and POEGMA gelation to develop a new nanocomposite injectable hydrogel platform with readily tailorable anisotropic properties that can better mimic native muscle tissue microenvironments and direct the orientation of differentiated skeletal muscle myotubes in situ.

\section{Preparation and Characterization of Injectable Anisotropic Nanocomposite Hydrogels.} CNCs were mixed at various concentrations $(0.2-1.65 \mathrm{wt} \%)$ with solutions of both hydrazide and aldehyde-functionalized POEGMA and were subsequently loaded into either side of a double barrel syringe. The precursor mixtures were extruded into molds (for hydrogel physical characterization), glass capillaries (for small angle x-ray scattering, SAXS) or tissue culture wells (for cell growth and differentiation) on a benchtop and allowed to gel either without any intervention or following placement between the poles of a rare earth magnet, as shown schematically in Figure 1. 


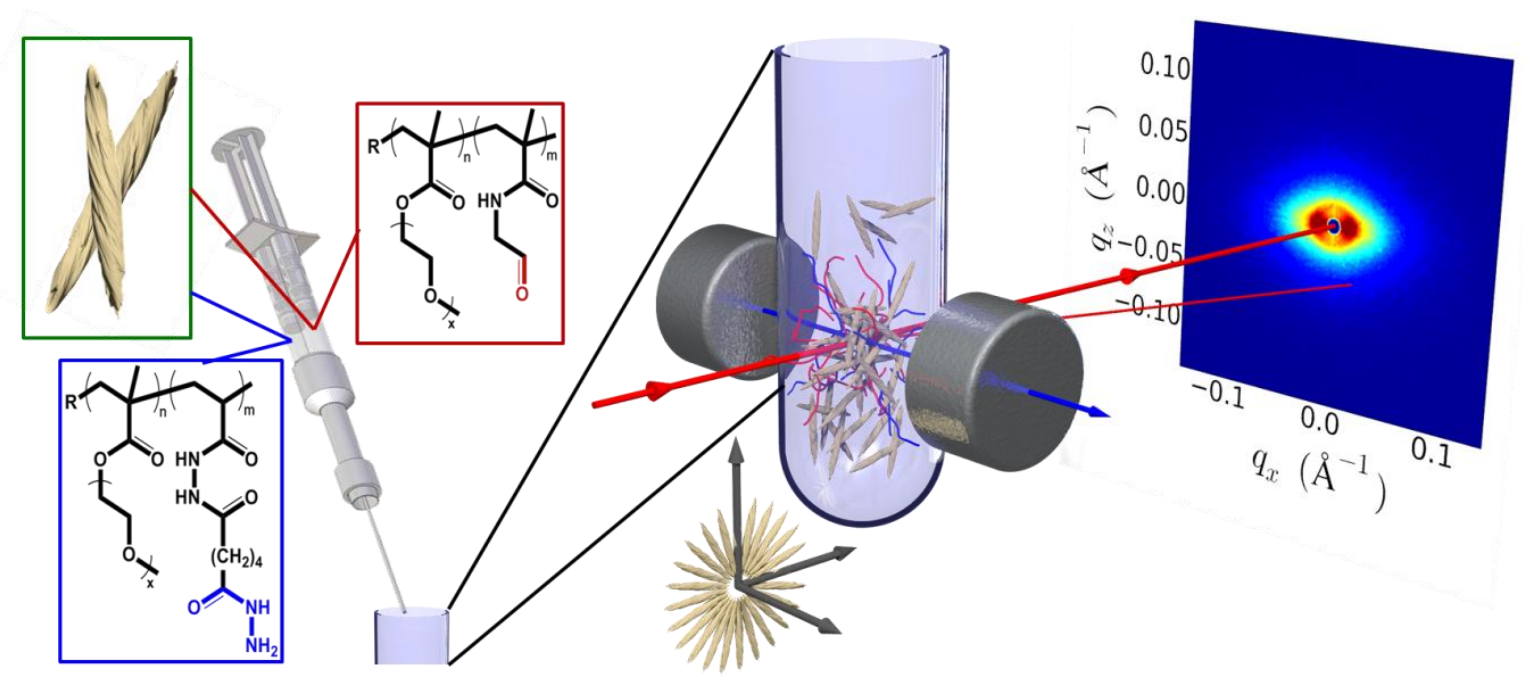

Figure 1. Schematic representation of injectable CNC-POEGMA nanocomposite hydrogels. After reactive coextrusion, the mixture was placed within a magnetic field to align CNCs inside the POEGMA hydrogel network. Alignment is demonstrated via small angle X-ray scattering whereby the x-ray beam (red) probes alignment perpendicular to the magnetic field direction (blue); a characteristic anisotropic scattering pattern is shown on an area detector.

The gelation times were unaffected by the application of a magnetic field and ranged from 10 minutes (1.65 wt\% CNC) to 25 minutes (0.2 wt\% CNC). Regardless, all nanocomposite hydrogels were allowed to gel for at least 1 hour before further testing or characterization. SAXS data show strong interactions between CNCs and POEGMA within the nanocomposite hydrogel whereby both POEGMA solvation and long-range CNC ordering are suppressed compared to pure POEGMA or CNC phases (Supporting Information, Figure S1). Figure 2 shows SAXS angular linecuts through the low- $q$ scattering region (a region dominated by the $\mathrm{CNC}$ form factor) of the $1.65 \mathrm{wt} \%$ CNC-POEGMA nanocomposite hydrogel in the presence and absence of an external magnetic field; linecuts for all other hydrogels are shown in Supporting Information, Figure S2. If no magnetic field is applied (Figure 2A), the resulting hydrogel composite is isotropic (i.e., no preferential CNC orientation is observed). However, exposure of the in situ gelling nanocomposite hydrogel to a $1.2 \mathrm{~T}$ magnetic field (Figure 2B) induces CNC 
orientation/anisotropy across a broad range of $q$ values. This result implies that the CNCs have been oriented by the magnetic field, despite the presence of the rapidly crosslinking POEGMA network, and that the orientation persists when the magnetic field is removed.
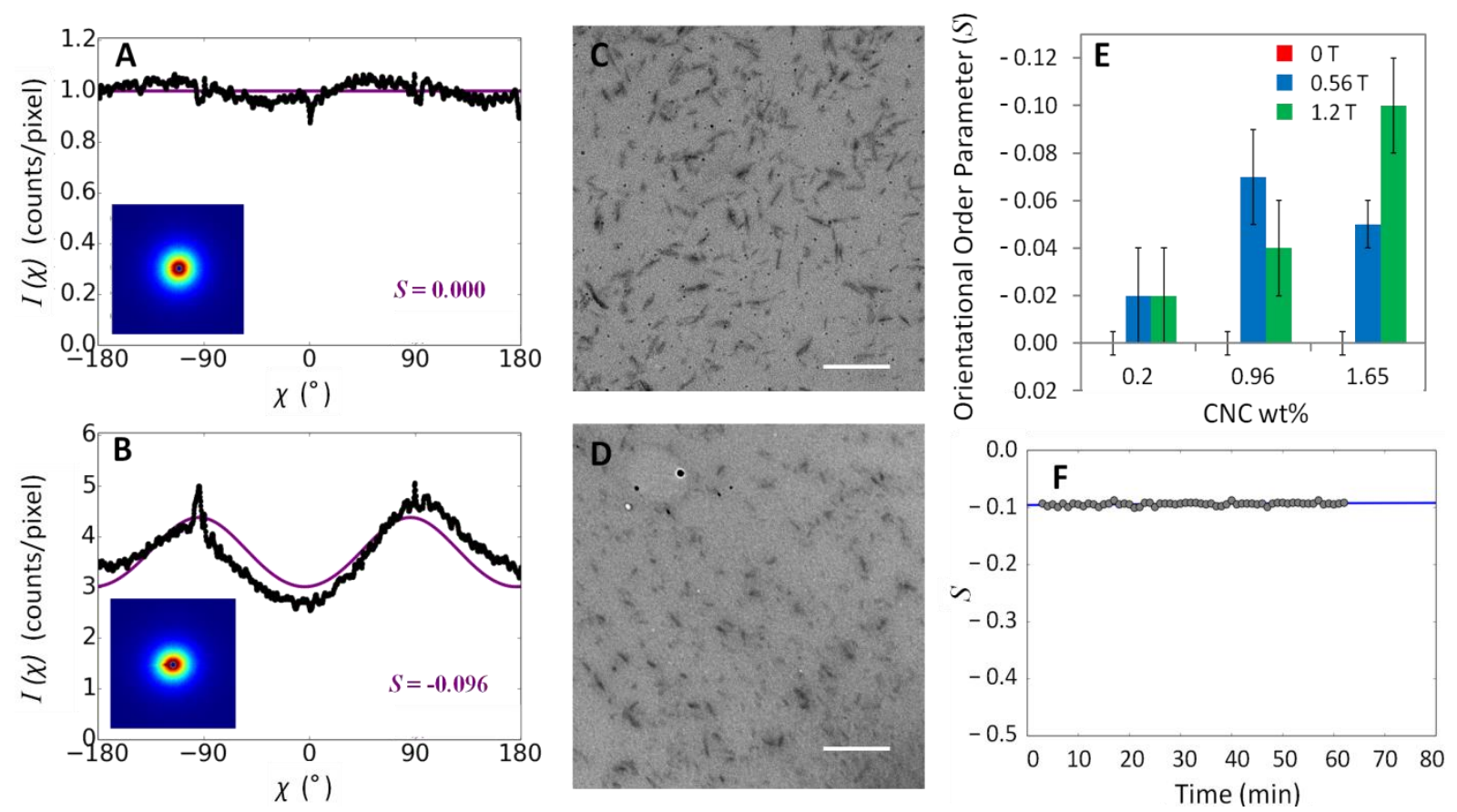

Figure 2. (A, B) Small angle $x$-ray scattering angular linecuts for $1.65 \mathrm{wt} \%$ CNC-POEGMA nanocomposite hydrogels (A) not exposed to a magnetic field during gelation and (B) exposed to a 1.2 T magnetic field during gelation. Linecuts were obtained in a representative region of the low- $q$ scattering regime $\left(0.05 \AA^{-1}\right) 1$ hour post-gelation. Insets show characteristic scattering patterns as displayed on an area detector. (C, D) Cryo-sectioned TEM images (sectioned perpendicular to the applied magnetic field) for a $1.65 \mathrm{wt} \%$ CNC-POEGMA nanocomposite hydrogel (C) not exposed to a magnetic field during gelation and (D) exposed to a $1.2 \mathrm{~T}$ magnetic field during gelation. Scale bars $=1 \mu \mathrm{m}$. (E) Orientational order parameter $(S$ ), calculated from SAXS data, as a function of CNC loading and applied magnetic field strength. (F) Kinetics of CNC alignment for $1.65 \mathrm{wt} \%$ CNC-POEGMA nanocomposite hydrogel exposed to a $1.2 \mathrm{~T}$ magnetic field; $S$ rapidly reaches its maximum absolute value (before the first data point is collected at 2 minutes) and remains constant over time.

TEM images comparing the isotropic nanocomposite hydrogel (Figure 2C) and the magneticallyaligned anisotropic nanocomposite hydrogel (Figure 2D) suggest moderate anti-alignment of the CNCs when gelation is conducted in a magnetic field. The degree of anisotropy $(S)$, calculated 
from the SAXS data using our previously-established protocol ${ }^{74}$ and varying between 0 for a perfectly isotropic system and -0.5 for a perfectly anisotropic system, increases as the CNC concentration and/or the magnetic field strength increase (Figure 2E); this result is consistent with alignment trends observed in pure CNC suspensions. ${ }^{74}$ For the hydrogel with a loading of $1.65 \mathrm{wt} \%$ CNCs exposed to a $1.2 \mathrm{~T}$ field (Figure 2B), an order parameter of $S \approx-0.1$ was calculated, indicating moderate anti-alignment in which the CNC long axis is aligned perpendicular to the magnetic field. See Supporting Information, Additional Results section for a full discussion and comparison of these results to those observed for CNC suspensions alone.

To gain insight into the timescale of CNC alignment in POEGMA hydrogels, we further studied the kinetics of magnetic alignment by performing SAXS in situ by extruding the hydrogel directly into a SAXS sample holder exposed to a static magnetic field. Pure CNC suspensions exhibit two timescales for alignment in similar magnetic fields: a rapid $(<2$ minute) onset of substantial alignment followed by a slower ( 30 minute) cooperative ordering that further increases the orientational order parameter. ${ }^{74}$ However, as demonstrated in Figure $2 \mathrm{~F}$, the nanocomposite hydrogels exhibit only the first of these processes. The overall scattering intensity decreases over time (Supporting Information, Figure S2), indicative of a gelation process that disrupts $\mathrm{CNC}$ packing; note that the time constant of $~ 16$ minutes measured from Figure $\mathrm{S} 2$ is on a similar order of magnitude as the $\sim 10$ minute macroscopic gelation time that governs CNC mobility in the matrix. However, the orientational order parameter rapidly saturates ( $<2$ minutes) to its maximum absolute value. This result suggests that very weak crosslinking or even the formation of local pre-gel domains is sufficient to "lock in" the CNC alignment even when the magnetic field is removed. Thus, the ultimate degree of alignment is limited not by intrinsic CNC response or cooperative ordering but rather by the timescale of the 
initial polymer-CNC association/network gelation. The combination of hydrazone POEGMA chemical crosslinks and CNC-POEGMA physical crosslinks prevent further magnetic orientation once the gel is set.

Physical Properties of Hydrogels with Magnetically Aligned CNCs. Magnetic alignment induces significant changes in a range of hydrogel physical properties. Figure 3 shows the swelling, degradation, and mechanical responses of hydrogels prepared with three different CNC concentrations in the presence and absence of a $0.56 \mathrm{~T}$ magnetic field. No significant difference in swelling was observed when $0.2 \mathrm{wt} \% \mathrm{CNCs}$ were incorporated into the hydrogel whether or not a magnetic field was applied, consistent with the small amount of incorporated CNCs and the minimal amount of alignment observed (Figure 2E). However, at both 0.96 and $1.65 \mathrm{wt} \% \mathrm{CNC}$ loadings, magnetically aligned hydrogels swelled significantly more than the corresponding isotropic gels. We attribute this result to the increased planar alignment of CNCs in the direction perpendicular to the applied field, allowing for increased POEGMA swelling (and lower POEGMA-CNC physical interactions) in the direction parallel to the field. 

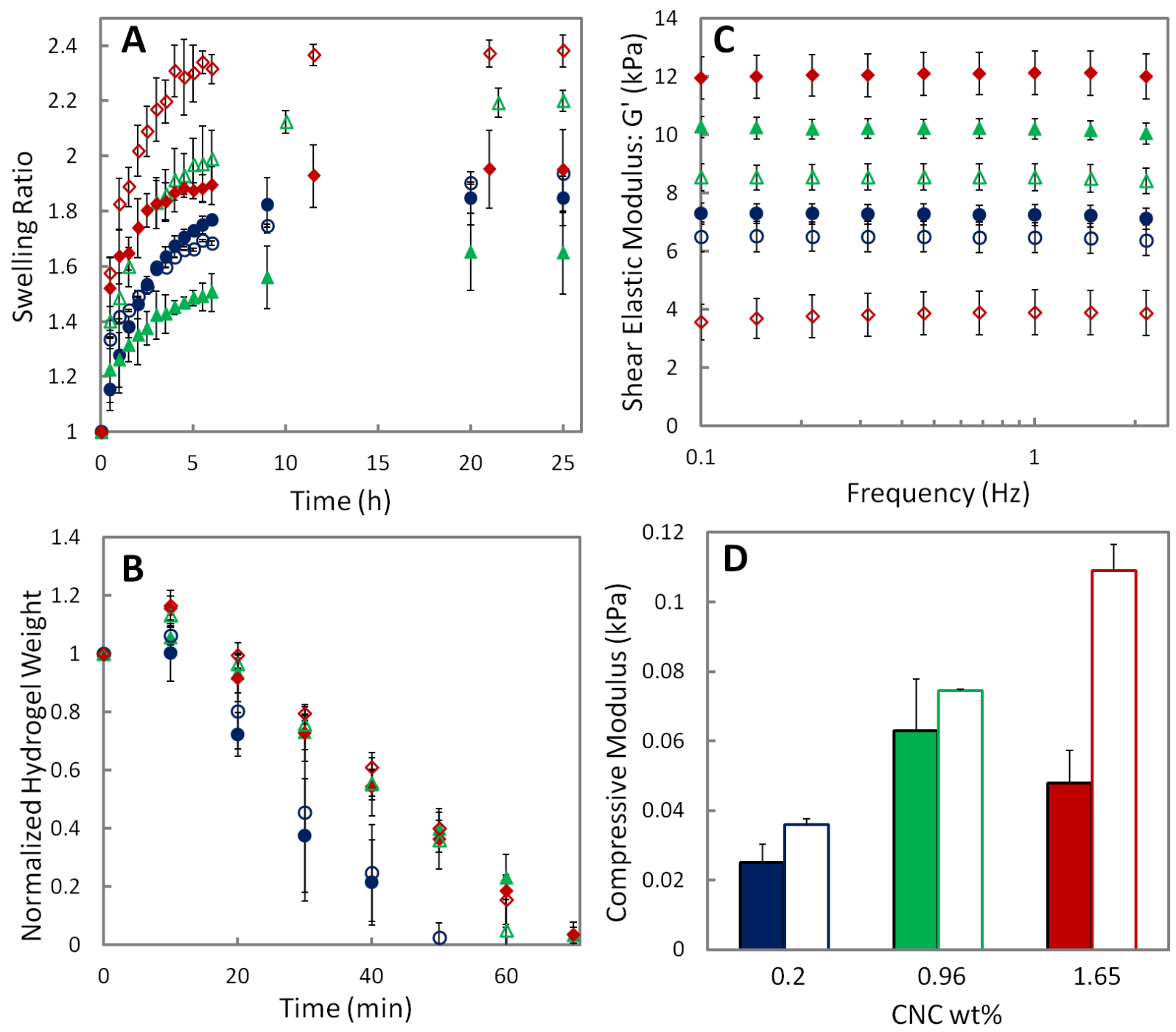

Figure 3. Physical properties of CNC-POEGMA hydrogels prepared with and without a magnetic field: (A) swelling (in $10 \mathrm{mM}$, pH 7.4 PBS buffer), (B) accelerated degradation (in 0.1 $\mathrm{M} \mathrm{HCl}$ ), (C) shear modulus parallel to the direction of CNC alignment, and (D) compressive modulus perpendicular to the direction of $\mathrm{CNC}$ alignment profiles for magnetically aligned (unfilled) and control (filled) hydrogels with 0.2 (blue $\circ$ ), 0.96 (green $\Delta$ ) or $1.65 \mathrm{wt} \%$ (red $\diamond$ ) CNC loading.

In contrast, $\mathrm{CNC}$ alignment showed no significant effect on hydrogel degradation in $0.1 \mathrm{M} \mathrm{HCl}$ (Figure 3B), although the presence of CNCs (either aligned or un-aligned) decreased the rate of hydrogel degradation relative to a POEGMA gel alone due to the increased physical crosslinking between POEGMA and CNCs. ${ }^{34}$ Hydrogel degradation is primarily driven by the hydrolytic 
cleavage of hydrazone crosslinks, ${ }^{30}$ which based on the result in Figure 3B appear to be equally accessible in hydrogels with aligned or unaligned CNCs.

Clear evidence for, and effects of, $\mathrm{CNC}$ alignment is also apparent in the mechanical properties of the hydrogel nanocomposites. While the random incorporation of CNCs into POEGMA hydrogels leads to isotropic increases in measured modulus values ${ }^{34}$ (indeed among the highest reported for injectable hydrogels), magnetic alignment of the CNCs results in anisotropic mechanical responses relative to the direction of $\mathrm{CNC}$ alignment. Shear testing performed in a radial direction perpendicular to the original direction of the applied magnetic field during gelation (i.e., CNCs are aligned parallel to the applied stress) indicated that the shear elastic modulus $G^{\prime}$ was significantly lower in hydrogels with aligned CNCs versus the corresponding isotropic controls (Figure 3C). The planar alignment of CNCs in the shear plane correlates to fewer CNCs perpendicular to the direction of shear and thus fewer CNC-CNC interactions, leading to weaker mechanics along this axis. The decrease in $G^{\prime}$ upon CNC alignment was significantly more pronounced as the CNC concentration increased from $0.2 \mathrm{wt} \%(1.1 \pm 0.1$-fold decrease in $G^{\prime}$ upon alignment) to $1.65 \mathrm{wt} \%$ (3.2 \pm 0.2 -fold decrease in $G^{\prime}$ upon alignment), consistent with the increase in overall anisotropy as the $\mathrm{CNC}$ concentration increases (Figure 2E). In contrast, the hydrogel compressive modulus (tested at $25 \%$ compression in a direction parallel to the applied external magnetic field, such that $\mathrm{CNCs}$ are aligned perpendicular to the applied stress) significantly increased upon CNC alignment (Figure 3D). Here, closely packed planes of aligned rigid CNCs perpendicular to the direction of compression provide increased resistance to compression at high hydrogel deformation. The magnitude of this increase is again a function of the $\mathrm{CNC}$ content, with the relative compressive modulus enhancement upon alignment increasing as the CNC content is increased from $0.2 \mathrm{wt} \%(1.4 \pm 0.2$-fold increase in 
compressive modulus upon magnetic alignment) to $1.65 \mathrm{wt} \%(2.3 \pm 0.2$-fold increase in compressive modulus upon magnetic alignment).

\section{Directing Muscle Cell Growth and Differentiation with Injectable Anisotropic Hydrogels.}

The effect of magnetic alignment on myoblast terminal differentiation was studied through both 2D and 3D experiments using hydrogels with high CNC loadings (1.65 wt $\%$ ), as these gels exhibit the highest degree of alignment (Figure 2E). For the 2D experiments, cells were plated on top of hydrogel constructs gelled either in the presence or absence of a $0.56 \mathrm{~T}$ magnetic field. After two days of culture, no apparent differences were observed in myoblast morphology or orientation between magnetically aligned and control hydrogels (Figure 4A), with cells remaining viable and spread over all tested surfaces. Following, differentiation media was added to promote differentiation of the myoblasts to myotubes over an additional eight-day incubation period (Figure 4B). Multinuclear myotubes were formed on all surfaces, and their alignment was quantitatively determined through image analysis (Figure 4C). ${ }^{43,75}$ For each substrate, at least four images were analyzed and plotted on a histogram to show the predominant direction of myotube alignment (orientation angle $=0^{\circ}$ ) and the deviation from that angle (Figure 4B; see Supporting Information, Figure S4 for additional images covering a larger field of view). The high angle deviation and flat distribution of angles observed on both the TCPS control and the unaligned hydrogels indicated only minimal myotube orientational order on both interfaces (Figure 4D, left and middle). In contrast, myotubes cultured on aligned CNC-POEGMA hydrogels exhibited a highly oriented morphology, as indicated by the much lower $25.0 \pm 4.5^{\circ}$ angle deviation observed (Figure 4D, right). 

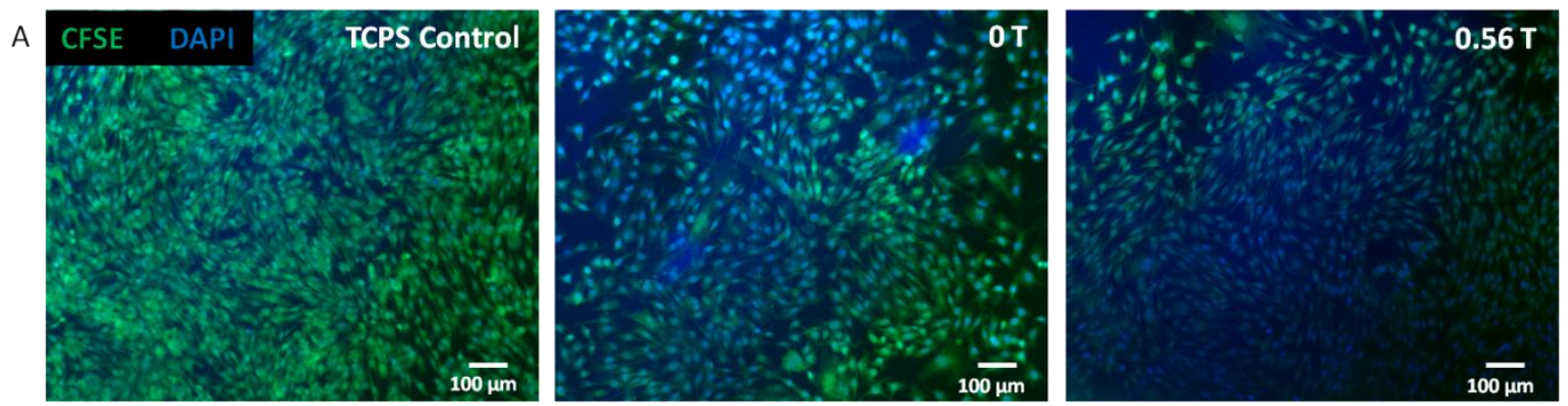

B
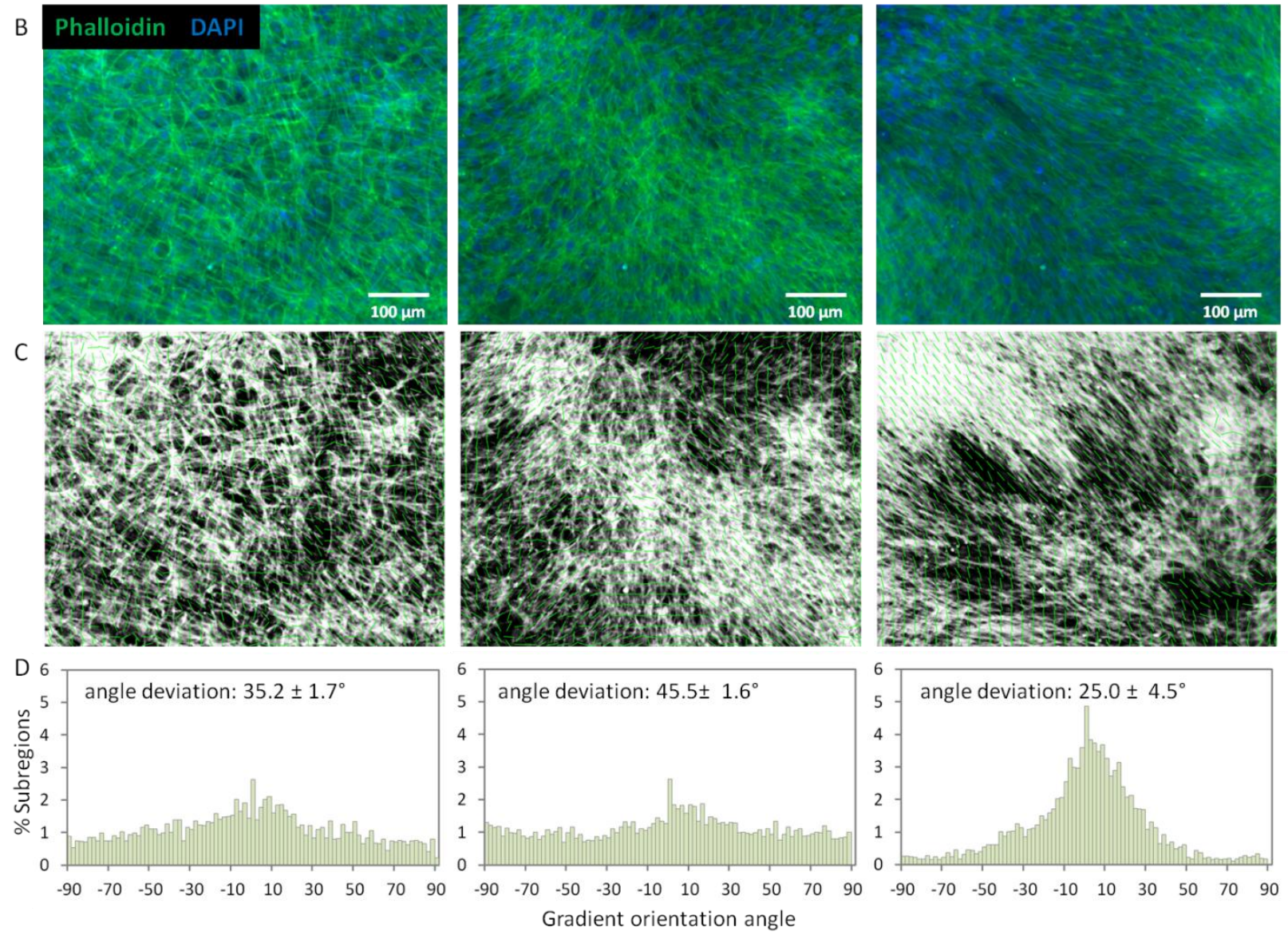

Figure 4. Effects of $\mathrm{CNC}$ alignment on directing cell growth (20x magnification): (A) $\mathrm{C} 2 \mathrm{C} 12$ mouse myoblasts cultured on TCPS control, unaligned, and aligned hydrogels for 48 hours. (B) Differentiated myotubes on TCPS, unaligned, and aligned hydrogels after culture in differentiation media for subsequent eight days. (C) Image analysis on myotube F-actin filaments from the images in panel B, and (D) histograms of their orientational order on TCPS, aligned, and unaligned hydrogels. Histograms are compiled from at least four images for each condition (TCPS control, unaligned, and aligned gels).

Note that a control experiment in which myoblast cells were plated on tissue culture polystyrene, exposed to the same $0.56 \mathrm{~T}$ magnetic field for 1 hour, and subsequently grown and differentiated 
using the same protocols showed no significant difference in cell alignment relative to cells not exposed to the magnetic field ( $p>0.73$, Supporting Information Figure S6). Therefore, the increase in cell alignment observed with the magnetically aligned hydrogels can be attributed solely to $\mathrm{CNC}$ alignment within the gel and not to the interaction of the magnetic field with the cells directly.

The capacity of the anisotropic injectable hydrogels to facilitate alignment in 3D was subsequently tested by suspending $\mathrm{C} 2 \mathrm{C} 12$ myoblasts within both the hydrazide- and aldehydefunctionalized precursor polymer solutions and loading them into separate barrels of a doublebarrel syringe. Gels were then extruded in the presence or absence of a magnetic field (as previously described for the physical experiments) and incubating using the same differentiation protocol used for the $2 \mathrm{D}$ experiments. The encapsulated cells remained viable over the duration of the experiment (Figure 5A) and were noted to significantly accelerate the degradation of the hydrogel, which exhibited substantial decreases in mass during the last two media replacement steps (i.e. on days 6 and 8 of the differentiation cycle). As such, while the hydrogel matrix assists in aligning the cells earlier in the protocol, as the cells grow they can subsequently create their own extracellular matrix to replace the initial scaffold but retain those alignment cues. Image analysis (Figure 5B) shows a more random orientational distribution for myotubes cultured on TCPS or within unaligned gels but significantly higher orientation order for myotubes cultured within aligned gels (Figure 5C), observations maintained even when a wider field of view was considered (Supporting Information, Figure S5). 

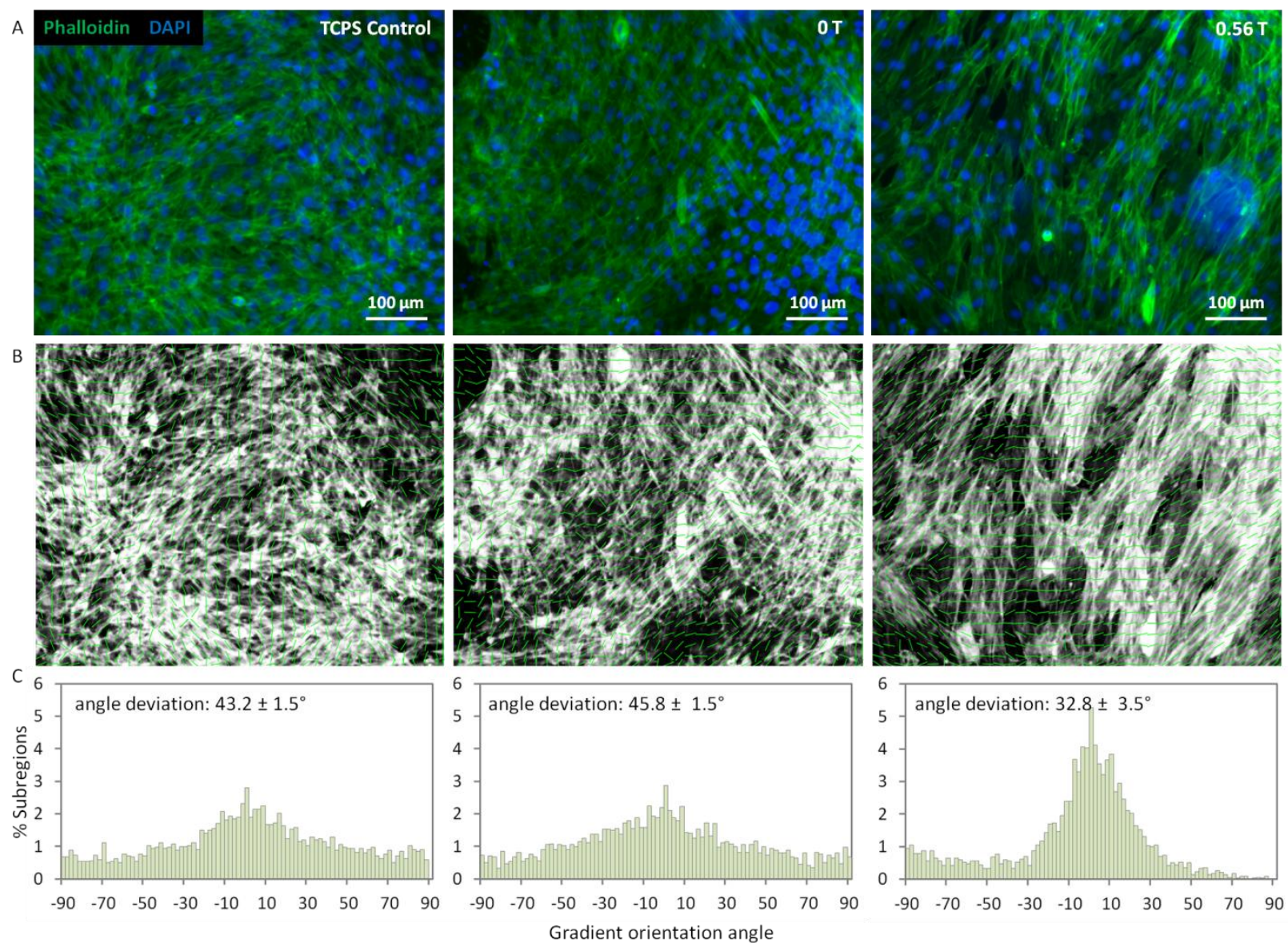

Figure 5. Effects of CNC alignment on directing cell differentiation in 3D (20x magnification): (A) Differentiated C2C12 mouse myotubes cultured on TCPS, and within unaligned, and aligned hydrogels. (B) Image analysis on myotube F-actin filaments from the images in panel A, and (C) histograms of their orientational order on TCPS, aligned, and unaligned hydrogels. Histograms are compiled from at least four images for each condition (TCPS control, unaligned, and aligned gels).

The ability to readily align $\mathrm{CNCs}$ within injectable and mechanically durable nanocomposite hydrogels that can in situ crosslink following injection suggests the potential of magneticallyaligned CNC-POEGMA hydrogels as a platform for developing minimally invasive structured materials for functional tissue engineering applications, including biomimicry of highly anisotropic muscle tissue. ${ }^{76,77}$ We note that effective cell alignment occurs even though the dimensions of the CNCs that promote the alignment $(\sim 10 \mathrm{~nm}$ cross-section $\times 150 \mathrm{~nm}$ length) 
are roughly two orders of magnitude smaller than the cells that they are aligning $(\sim 10-20 \mu \mathrm{m}$ minimum), showing for the first time the potential of large-scale nanomaterial orientation for directing cell behavior in 3D. We hypothesize that this works based on a combination of CNCmediated contact guidance (shown previously to direct cell orientation on spin coated CNC thin films), ${ }^{67,68}$ and the hydrogel mechanical differences perpendicular and parallel to the aligned CNC direction that make it easier for cells to spread and expand parallel to the aligned CNC planes (Figs. 3C and 3D). Although there is some evidence in the literature suggesting that adherent muscle cells will self-align in response to an external magnetic field, these effects have only been observed at field strengths greater than $10 \mathrm{~T}^{78,79}$ or under continual exposure to the field over multiple days. ${ }^{80}$ In this work, the magnetic field was much weaker $(0.56 \mathrm{~T})$ and much shorter in duration (one time for one hour), with a cell-only control (Fig. S6) showing no significant direct magnetic field effects on cell alignment. As such, the ultimate alignment of the cells can only be attributable to the properties of the hydrogel rather than any direct cell-magnet interactions. It should also be noted that the degree of alignment achieved in $2 \mathrm{D}$ mirrors that obtained on spin coated $\mathrm{CNC}$ thin films (angle deviation $\approx 28^{\circ}$ ); ${ }^{68}$ furthermore, the $3 \mathrm{D}$ alignment achieved is comparable to that demonstrated on highly controlled physically templated hydrogels $\left(\text { angle deviation }=25 \pm 1^{\circ}\right)^{43,75}$ and approaches those observed on microchannelled substrates (angle deviation $\left.<10^{\circ}\right){ }^{41,42}$ Notably, any of these previously reported physical templating approaches are either inappropriate for direct in vivo application or would require surgical delivery for clinical use, making the CNC-POEGMA system of particular promise for minimally invasive muscle tissue engineering. The magnitude of magnetic field applied herein for CNC alignment is also easily achievable with existing clinical magnetic resonance imaging (MRI) systems, ${ }^{81}$ offering translational potential for growing aligned tissue in vivo. 
In summary, we have demonstrated an injectable nanocomposite hydrogel platform with tunable nanoscale anisotropy facilitated by the application of an external magnetic field. CNCs were shown to align in situ on the order of minutes within a rapidly gelling chemically crosslinked POEGMA hydrogel matrix that subsequently "locks" the magnet-induced CNC alignment in place. Hydrogels containing aligned CNCs exhibit significantly higher swelling, lower shear moduli, and higher compressive moduli relative to isotropic CNC-POEGMA hydrogels despite having identical chemical compositions. Furthermore, CNC alignment leads to a significant improvement in myotube orientation both on (2D) and within (3D) the hydrogels. We anticipate that this functional anisotropic injectable hydrogel platform will be particularly relevant for engineering oriented tissues (e.g., muscle, cartilage, and nerve) by remotely inducing the required anisotropy in the scaffold using a safe and fully non-invasive magnetic field stimulus following simple injection, thus avoiding the need for surgical procedures.

\section{ASSOCIATED CONTENT}

The Supporting Information is available free of charge via the Internet at http://pubs.acs.org. Experimental details, small angle $\mathrm{x}$-ray scattering analysis of CNC-POEGMA nanocomposite hydrogels, discussion of hydrogel SAXS observations and CNC alignment in hydrogels at low concentrations, SAXS linecuts for all hydrogel samples, additional fluorescence images/image analysis on differentiated $\mathrm{C} 2 \mathrm{C} 12$ myoblasts in 2D and 3D, magnetic control experiments for $\mathrm{C} 2 \mathrm{C} 12$ alignment. 


\section{AUTHOR INFORMATION}

\section{Corresponding Author}

*E-mail: hoaretr@mcmaster.ca

\section{Author Contributions}

KD led the design of the experiments, performed the majority of the experimental work, and wrote the bulk of the manuscript. KY assisted with running and analyzing the SAXS data, KJWC assisted with the physical characterization experiments, BC performed the image analysis to confirm myotube alignment, and EC and TH provided guidance in the experimental design and refined the manuscript text. All authors have given approval to the final version of the manuscript.

\section{Notes}

The authors declare no competing financial interest.

\section{ACKNOWLEDGMENTS}

Funding from the Natural Sciences and Engineering Research Council of Canada (Discovery Grants RGPIN 356609 and 402329) as well as NSERC CREATE-IDEM (Integrated Design of Extracellular Matrices, grant 398058) is gratefully acknowledged. We thank Yugang Zhang and Dmytro Nykypanchuk for assistance with the in situ magnetic cell, and Peter Jonasson and Christopher Mitchelitis for use of the $0.56 \mathrm{~T}$ rare earth magnet. This research used resources of the Center for Functional Nanomaterials, which is a U.S. DOE Office of Science Facility, at Brookhaven National Laboratory under Contract No. DE-SC0012704. 


\section{REFERENCES}

(1) Hoare, T. R.; Kohane, D. S. Polymer 2008, 49 (8), 1993-2007.

(2) Gaharwar, A. K.; Peppas, N. A.; Khademhosseini, A. Biotechnol. Bioeng. 2014, 111 (3), $441-453$.

(3) Hoffman, A. S. Adv. Drug Deliv. Rev. 2002, 54 (1), 3-12.

(4) Yu, L.; Ding, J. Chem. Soc. Rev. 2008, 37 (8), 1473-1481.

(5) Khademhosseini, A.; Vacanti, J. P.; Langer, R. Sci. Am. 2009, 300, 64-71.

(6) Gaharwar, A. K.; Dammu, S. A.; Canter, J. M.; Wu, C. J.; Schmidt, G. Biomacromolecules 2011, 12 (5), 1641-1650.

(7) Johnson, T. D.; Christman, K. L. Expert Opin. Drug Deliv. 2013, 10 (1), 59-72.

(8) Farnebo, S.; Woon, C. Y. L.; Schmitt, T.; Joubert, L.-M.; Kim, M.; Pham, H.; Chang, J. Tissue Eng. Part A 2014, 20 (9-10), 1550-1561.

(9) Paul, A.; Hasan, A.; Kindi, H. Al; Gaharwar, A. K.; Rao, V. T. S.; Nikkhah, M.; Shin, S. R.; Krafft, D.; Dokmeci, M. R.; Shum-Tim, D.; Khademhosseini, A. ACS Nano 2014, 8 (8), 8050-8062.

(10) Knipe, J. M.; Peppas, N. A. Regen. Biomater. 2014, 1 (1), 57-65.

(11) He, C.; Kim, S. W.; Lee, D. S. J. Control. Release 2008, 127 (3), 189-207.

(12) Kretlow, J. D.; Klouda, L.; Mikos, A. G. Adv. Drug Deliv. Rev. 2007, 59 (4-5), 263-273.

(13) Du, A. W.; Stenzel, M. H. Biomacromolecules 2014, 15 (4), 1097-1114.

(14) Guvendiren, M.; Lu, H. D.; Burdick, J. A. Soft Matter 2012, 8 (2), 260-272.

(15) Klier, J.; Scranton, A. B.; Peppas, N. A. Macromolecules 1990, 23 (23), 4944-4949.

(16) Kopeček, J.; Yang, J. Acta Biomater. 2009, 5 (3), 805-816.

(17) Nguyen, K. T.; West, J. L. Biomaterials 2002, 23, 4307-4314.

(18) Lai, J. Y. J. Mater. Sci. Mater. Med. 2010, 21 (6), 1899-1911.

(19) Balakrishnan, B.; Jayakrishnan, A. Biomaterials 2005, 26 (18), 3941-3951.

(20) Nimmo, C. M.; Shoichet, M. S. Bioconjug. Chem. 2011, 22 (11), 2199-2209.

(21) Malkoch, M.; Vestberg, R.; Gupta, N.; Mespouille, L.; Dubois, P.; Mason, A. F.; Hedrick, J. L.; Liao, Q.; Frank, C. W.; Kingsbury, K.; Hawker, C. J. Chem. Commun. 2006, No. 26, 2774-2776. 
(22) Nimmo, C. M.; Owen, S. C.; Shoichet, M. S. Biomacromolecules 2011, 12 (3), 824-830.

(23) Shu, X. Z.; Liu, Y.; Luo, Y.; Roberts, M. C.; Prestwich, G. D. Biomacromolecules 2002, 3 (6), 1304-1311.

(24) Ossipov, D. A.; Piskounova, S.; Varghese, O. P.; Hilborn, J. Biomacromolecules 2010, 11 (9), 2247-2254.

(25) Fu, Y.; Kao, W. J. J. Biomed. Mater. Res. - Part A 2011, 98 A (2), 201-211.

(26) Saito, H.; Hoffman, A. S.; Ogawa, H. I. J. Bioact. Compat. Polym. 2007, 22 (6), 589-601.

(27) Ito, T.; Yeo, Y.; Highley, C. B.; Bellas, E.; Kohane, D. S. Biomaterials 2007, 28 (23), 3418-3426.

(28) Bakaic, E.; Smeets, N. M. B.; Hoare, T. RSC Adv. 2015, 5 (45), 35469-35486.

(29) Bakaic, E.; Smeets, N. M. B.; Dorrington, H.; Hoare, T. RSC Adv. 2015, 5 (42), 3336433376.

(30) Smeets, N. M. B.; Bakaic, E.; Patenaude, M.; Hoare, T. Chem. Commun. 2014, 50 (25), 3306-3309.

(31) Smeets, N. M. B.; Bakaic, E.; Patenaude, M.; Hoare, T. Acta Biomater. 2014, 10 (10), 4143-4155.

(32) Smeets, N. M. B.; Bakaic, E.; Yavitt, F. M.; Yang, F.; Rheinsta, M. C.; Hoare, T. Macromolecules 2014.

(33) Smeets, N. M. B.; Patenaude, M.; Kinio, D.; Yavitt, F. M.; Bakaic, E.; Yang, F.-C.; Rheinstädter, M.; Hoare, T. Polym. Chem. 2014, 5, 6811.

(34) De France, K. J.; Chan, K. J. W.; Cranston, E. D.; Hoare, T. Biomacromolecules 2016, 17 (2), 649-660.

(35) Melchels, F. P. W.; Domingos, M. A. N.; Klein, T. J.; Malda, J.; Bartolo, P. J.; Hutmacher, D. W. Prog. Polym. Sci. 2012, 37 (8), 1079-1104.

(36) Kwee, B. J.; Mooney, D. J. Curr. Opin. Biotechnol. 2017, 47, 16-22.

(37) Neal, D.; Sakar, M. S.; Bashir, R.; Chan, V.; Asada, H. H. Tissue Eng. Part A 2015, 21 (11-12), 1848-1858.

(38) Neal, D.; Sakar, M. S.; Ong, L.-L. S.; Harry Asada, H. Lab Chip 2014, 14 (11), 1907.

(39) Kang, H.-W.; Lee, S. J.; Ko, I. K.; Kengla, C.; Yoo, J. J.; Atala, A. Nat. Biotechnol. 2016, $34(3), 312-319$.

(40) Mozetic, P.; Maria Giannitelli, S.; Gori, M.; Trombetta, M.; Rainer, A. J. Biomed. Mater. 
Res. Part A 2017.

(41) Huang, N. F.; Lee, R. J.; Li, S. Am. J. Transl. Res. 2010, 2 (1), 43-55.

(42) Huang, N. F.; Patel, S.; Thakar, R. G.; Wu, J.; Hsiao, B. S.; Chu, B.; Lee, R. J.; Li, S. Nano Lett. 2006, 6 (3), 537-542.

(43) Bian, W.; Liau, B.; Badie, N.; Bursac, N. Nat. Protoc. 2009, 4 (10), 1522-1534.

(44) Sicari, B. M.; Rubin, J. P.; Dearth, C. L.; Wolf, M. T.; Ambrosio, F.; Boninger, M.; Turner, N. J.; Weber, D. J.; Simpson, T. W.; Wyse, A.; Brown, E. H. P.; Dziki, J. L.; Fisher, L. E.; Brown, S.; Badylak, S. F. Sci. Transl. Med. 2014, 6 (234), 234ra58-234ra58.

(45) Pumberger, M.; Qazi, T. H.; Ehrentraut, M. C.; Textor, M.; Kueper, J.; StoltenburgDidinger, G.; Winkler, T.; von Roth, P.; Reinke, S.; Borselli, C.; Perka, C.; Mooney, D. J.; Duda, G. N.; Gei??ler, S. Biomaterials 2016, 99, 95-108.

(46) Sadtler, K.; Estrellas, K.; Allen, B. W.; Wolf, M. T.; Fan, H.; Tam, A. J.; Patel, C. H.; Luber, B. S.; Wang, H.; Wagner, K. R.; Powell, J. D.; Housseau, F.; Pardoll, D. M.; Elisseeff, J. H. Science 2016, 352 (6283), 366-370.

(47) Cezar, C. A.; Roche, E. T.; Vandenburgh, H. H.; Duda, G. N.; Walsh, C. J.; Mooney, D. J. Proc. Natl. Acad. Sci. 2016, 113 (6), 1534-1539.

(48) Voge, C. M.; Kariolis, M.; MacDonald, R. A.; Stegemann, J. P. J. Biomed. Mater. Res. Part A 2008, 86 (1), 269-277.

(49) Osorio-Madrazo, A.; Eder, M.; Rueggeberg, M.; Pandey, J. K.; Harrington, M. J.; Nishiyama, Y.; Putaux, J.-L.; Rochas, C.; Burgert, I. Biomacromolecules 2012, 13 (3), $850-856$.

(50) Haque, M. A.; Kamita, G.; Kurokawa, T.; Tsujii, K.; Gong, J. P. Adv. Mater. 2010, 22 (45), 5110-5114.

(51) Yang, W.; Furukawa, H.; Gong, J. P. Adv. Mater. 2008, 20 (23), 4499-4503.

(52) Pei, X.; Zan, T.; Li, H.; Chen, Y.; Shi, L.; Zhang, Z. ACS Macro Lett. 2015, 4, 12151219.

(53) Walker, K. J.; Madihally, S. V. J. Biomed. Mater. Res. Part B 2015, 103 (6), 1149.

(54) Hockaday, L. A.; Kang, K. H.; Colangelo, N. W.; Cheung, P. Y. C.; Duan, B.; Malone, E.; Wu, J.; Girardi, L. N.; Bonassar, L. J.; Lipson, H.; Chu, C. C.; Butcher, J. T. Biofabrication 2012, 4 (3), 35005.

(55) Bai, H.; Polini, A.; Delattre, B.; Tomsia, A. P. Chem. Mater. 2013, 25 (22), 4551-4556. 
(56) Köhnke, T.; Elder, T.; Theliander, H.; Ragauskas, A. J. Carbohydr. Polym. 2014, 100, 2430.

(57) Barrow, M.; Zhang, H. Soft Matter 2013, 9 (9), 2723-2729.

(58) Yokoyama, F.; Achife, E. C.; Momoda, J.; Shimamura, K.; Monobe, K. Colloid Polym. Sci. 1990, $268(6), 552-558$.

(59) Mao, M.; He, J.; Liu, Y.; Li, X.; Li, D. Acta Biomater. 2012, 8 (6), 2175-2184.

(60) Chen, M.; Zhu, J.; Qi, G.; He, C.; Wang, H. Mater. Lett. 2012, 89, 8-11.

(61) Chau, M.; De France, K. J.; Kopera, B.; Machado, V. R.; Rosenfeldt, S.; Reyes, L.; Chan, K. J. W.; Förster, S.; Cranston, E. D.; Hoare, T.; Kumacheva, E. Chem. Mater. 2016, 28 (10), 3406-3415.

(62) Rose, J. C.; Camara-Torres, M.; Rahimi, K.; Köhler, J.; Moeller, M.; De Laporte, L. Nano Lett. 2017, 17 (6), 3782-3791.

(63) Discher, D. E.; Mooney, D. J.; Zandstra, P. W. Science 2009, 324 (5935), 1673-1677.

(64) Lin, N.; Dufresne, A. Eur. Polym. J. 2014, 59, 302-325.

(65) Moon, R. J.; Martini, A.; Nairn, J.; Simonsen, J.; Youngblood, J. Chem. Soc. Rev. 2011, $40(7), 3941-3994$.

(66) Habibi, Y.; Lucia, L. A.; Rojas, O. J. Chem. Rev. 2010, 110 (6), 3479-3500.

(67) Dugan, J. M.; Gough, J. E.; Eichhorn, S. J. Biomacromolecules 2010, 11 (9), 2498-2504.

(68) Dugan, J. M.; Collins, R. F.; Gough, J. E.; Eichhorn, S. J. Acta Biomater. 2013, 9 (1), 4707-4715.

(69) De France, K. J.; Hoare, T.; Cranston, E. D. Chem. Mater. 2017, 29 (11), 4609-4631.

(70) Frka-Petesic, B.; Sugiyama, J.; Kimura, S.; Chanzy, H.; Maret, G. Macromolecules 2015, 48 (24), 8844-8857.

(71) Sugiyama, J.; Chanzy, H.; Maret, G. Macromolecules 1992, 25 (16), 4232-4234.

(72) Frka-Petesic, B.; Jean, B.; Heux, L. Europhys. Lett. 2014, 107 (2), 28006.

(73) Abitbol, T.; Cranston, E. D. In Handbook of Green Materials; Oksman, K., Mathew, A. P., Bismarck, A., Rojas, O., Sain, M., Eds.; World Scientific, 2012; pp 79-103.

(74) De France, K. J.; Yager, K. G.; Hoare, T.; Cranston, E. D. Langmuir 2016, 32 (30), 7564 7571.

(75) Bian, W.; Bursac, N. Biomaterials 2009, 30 (7), 1401-1412.

(76) Qin, E. C.; Jugé, L.; Lambert, S. a; Paradis, V.; Sinkus, R.; Bilston, L. E. Radiology 2014, 
132661.

(77) Gennisson, J. L.; Deffieux, T.; Mace, E.; Montaldo, G.; Fink, M.; Tanter, M. Ultrasound Med. Biol. 2010, 36 (5), 789-801.

(78) Torbet, J.; Dickens, M. J. Fed. Eur. Biochem. Soc. Lett. 1984, 173 (2), 403-406.

(79) Iwasaka, M.; Miyakoshi, J.; Ueno, S. Vitr. Cell. Dev. Biol. - Anim. 2003, 39 (3-4), 120123.

(80) Coletti, D.; Teodori, L.; Albertini, M. C.; Rocchi, M.; Pristerà, A.; Fini, M.; Molinaro, M.; Adamo, S. Cytom. Part A 2007, 71 (10), 846-856.

(81) Haacke, E. M.; Brown, R. W.; Thompson, M. R.; Venkatesan, R. Magnetic resonance imaging: physical principles and sequence design; Wiley-Liss: New York, 1999. 